\title{
Sub-diffraction-limited imaging by photobleaching imprinting microscopy
}

Liang Gao, Alejandro Garcia-Uribe, Yan Liu, Chiye Li, Lihong Wang

Liang Gao, Alejandro Garcia-Uribe, Yan Liu, Chiye Li, Lihong Wang, "Subdiffraction-limited imaging by photobleaching imprinting microscopy," Proc. SPIE 8950, Single Molecule Spectroscopy and Superresolution Imaging VII, 895015 (4 March 2014); doi: 10.1117/12.2038573

SPIE. Event: SPIE BiOS, 2014, San Francisco, California, United States 


\title{
Sub-diffraction-limited imaging by photobleaching imprinting microscopy
}

\author{
Liang Gao, Alejandro Garcia-Uribe, Yan Liu, Chiye Li, and Lihong Wang $\dagger$ \\ Optical Imaging Laboratory, Department of Biomedical Engineering, Washington University in St. \\ Louis., St. Louis, MO 63130, USA \\ †LHWANG@WUSTL.EDU
}

\begin{abstract}
We present a generic sub-diffraction-limited imaging method - photobleaching imprinting microscopy (PIM) - for biological fluorescence imaging. A lateral resolution of $110 \mathrm{~nm}$ was measured, more than a two-fold improvement over the optical diffraction limit. Unlike other super-resolution imaging techniques, PIM does not require complicated illumination modules or specific fluorescent dyes. PIM is expected to facilitate the conversion of super-resolution imaging into a routine lab tool, making it accessible to a much broader biological research community.
\end{abstract}

Keywords: fluorescence microscopy, super-resolution microscopy

\section{INTRODUCTION}

Fluorescence microscopy has been extensively used to gain a deeper understanding of cell and tissue biology[1]. Despite widespread applications, fluorescence microscopy faces a fundamental bottleneck in its resolving capability - the optical diffraction limit, which was first stated by Ernst Abbe in 1873. Due to this limitation, the achievable spatial resolution of conventional fluorescence microscopy is $\sim 250 \mathrm{~nm}$ in the lateral direction, and $\sim 500 \mathrm{~nm}$ in the axial direction. Since many cellular organelles, such as microtubules, actin fibers, and ribosomes, are smaller than this size, breaking the optical diffraction limit has been the holy grail of light microscopy over the past several decades.

To address this challenge, a wide array of methodologies has been introduced, allowing the fine structures of a biological cell or tissue to be revealed at the super-resolution level. Depending on the conceptual shared thread, superresolution techniques are normally based on two distinct strategies. One strategy, referred to as probe-based superresolution, employs the stochastic nature of photoswitchable fluorophores, sequentially switching them on and off so that the signals from individual fluorescent molecules can be recorded consecutively. Merging all of the single-molecule centroid positions acquired by repeated cycles of photo-activation and photo-deactivation yields a final super-resolution image. Representative modalities using this strategy are stochastic optical reconstruction microscopy (STORM)[2], photoactivated localization microscopy (PALM) [3, 4], and photobleaching-based localization microscopy [5-7]. The second strategy, referred to as illumination-based super-resolution, utilizes non-linear optical approaches to modify and engineer the point-spread-function (PSF), resulting in a reduced focal spot size at the sample. Within this category, the most important techniques are stimulated emission depletion microscopy (STED)[8], structured illumination microscopy (SIM)[9], and saturated structured illumination microscopy (SSIM)[10]. Due to its superior resolving capability, superresolution imaging now has become an essential tool in examining a wide variety of biological molecules, pathways, and dynamics, providing unprecedented resolution at a scale down to tens of nanometers [11].

In spite of their revolutionary impact, most super-resolution techniques rely on specific fluorescent probes, e.g., PALM/STORM, or require complicated optical illumination modules, e.g., STED, limiting access by the general research community. To provide a generic method that can be readily implemented on a standard light microscope with conventional fluorescent dyes, here we present photobleaching imprinting microscopy (PIM) for super-resolution fluorescence imaging. Photobleaching is routinely encountered as a nuisance in fluorescence microscopy[12], which is leveraged here. PIM works by first imprinting a structured pattern onto the sample through photobleaching, followed by light interrogation with another focused Gaussian laser beam. The high-order components related to the laser fluence distribution can be extracted simply via a differential operation between two fluorescence intensities along time-lapse fluorescence decay. Using PIM, we demonstrated a lateral resolution of $\sim 110 \mathrm{~nm}$, more than a two-fold improvement over the optical diffraction limit. 


\section{PRINCIPLE}

Once a fluorophore is excited from the ground state to an excited state, part of the energy is released via radiative decay, emitting fluorescence with an intensity $I$ equal to

$$
I=C \mu_{a} F^{n}
$$

where $C$ is a constant, $\mu_{a}$ is the absorption coefficient of the fluorophore, $F$ is the laser fluence, and $n$ denotes the number of photons involved in an excitation event.

In fluorescence microscopy, photobleaching occurs when the excited electrons are trapped in a relatively long-lived triplet state. The forbidden triplet-singlet transition provides the fluorophore a much longer time to undergo irreversible chemical reactions with the environment than does the singlet-singlet transition [12]. The photobleaching of a fluorophore obeys an exponential temporal decay law

$$
\mu_{a}(t)=\mu_{a 0} \exp (-k t)
$$

where $t$ is the time, $\mu_{a 0}$ is the initial absorption coefficient of the fluorophore, and $k$ is the photobleaching rate. The photobleaching rate $k$ is a function of excitation laser fluence $F$, as described empirically by [13]

$$
k=B F^{m},
$$

where $B$ denotes a constant, and $m$ depicts the order of the dependence. For one-photon excitation fluorescence, $m=1$, while for two-photon excitation fluorescence, the photobleaching rate increases rapidly with $m \geq 3$ [13]. Combing Eqs. 1-3 gives

$$
I(t)=C \mu_{a 0} F^{n} \exp \left(-B F^{m} t\right)
$$

Subtracting the fluorescent intensity measured at a later time $t$ from the initial value measured at $t=0$ pixel by pixel yields a differential image with the following intensity:

$$
I_{\text {diff }}=C \mu_{a 0} F^{n}\left(1-\exp \left(-B F^{m} t\right)\right) .
$$

Equation 5 can be further Taylor expanded as

$$
I_{\text {diff }}=\sum_{l=1}^{\infty} D_{l} F^{m l+n}=D_{1} F^{m+n}+D_{2} F^{2 m+n}+\cdots
$$

Here $D_{l}=C \mu_{a 0}(-B t)^{l} / l$ !. In the case of one-photon excitation $(m=1, n=1)$, the dominating term in Eq. 6 is quadratic, i.e.,

$$
I_{d i f f}^{1 P} \propto F^{2} .
$$

In the case of two-photon excitation ( $m \geq 3, n=2$ ), the dominating term in Eq. 6 starts with an order $K$ larger than five, i.e.,

$$
I_{\text {diff }}^{2 P} \propto F^{K}, K \geq 5
$$

The nonlinearity depicted in Eq. 7 and 8 shrinks the PSF in all dimensions, thus enabling super-resolution imaging beyond the optical diffraction limit. 


\section{EXPERIMENTAL SETUP AND DATA PROCESSING}

The PIM experiments were implemented on a confocal laser scanning microscope equipped with a multi-photon imaging unit (FV1000, Olympus). Two continuous-wave laser sources (Argon and $\mathrm{He}-\mathrm{Ne}$ ) provide a total of 4 wavelengths (405 $\mathrm{nm}, 488 \mathrm{~nm}, 543 \mathrm{~nm}$, and $632 \mathrm{~nm}$ ) for one-photon excitation, and a Ti-sapphire femtosecond pulsed laser provides a tunable wavelength $(780 \mathrm{~nm}-1060 \mathrm{~nm})$ for two-photon excitation. The fluorescence was collected by a high NA microscope objective (PLAPON 60XO, NA = 1.4, for one-photon excitation; 25X MPE WATER IMMERSION, NA = 1.05 , for two-photon excitation) and was simultaneously detected by up to three separate PMT colour channels. The laser excitation and acquisition of fluorescence images were controlled by the accompanying microscope software (Fluoview, Olympus).

PIM is achieved by monitoring photobleaching-induced fluorescence decay, followed by a pixel-by-pixel differential operation between two consecutive fluorescent images. The PIM procedure can be performed by utilizing the time-lapse imaging module on a standard confocal microscope. To make surrounding regions unaffected at each sampling pixel, the scanning step size must be larger than the diffraction-limited PSF during acquiring a time-lapse dataset. Then we translate the sample a distance that is equal to the half of PIM's resolution and start the next cycle. The PIM images are calculated for each cycle and stitched together to form a final image.

Under the hypothesis that each fluorescence image captured along the time course is shot noise limited, the signal variance is correlated to the number of acquired photons $N$ by the relation

$$
\operatorname{var}\left(I_{f}\right)=N .
$$

The differential operation between $I_{f 1}$ and $I_{f 2}$ amplifies the variance by a factor of two, i.e.,

$$
\operatorname{var}\left(I_{\text {diff }}\right)=\operatorname{var}\left(I_{f 1}\right)+\operatorname{var}\left(I_{f 2}\right) \approx 2 \operatorname{var}\left(I_{f 1}\right)=2 N .
$$

The SNR of the resulting differential image thus equals

$$
S N R_{\text {diff }}=\frac{I_{f 1}-I_{f 2}}{\sqrt{\operatorname{var}\left(I_{d i f f}\right)}} \approx \frac{\Delta N}{\sqrt{2 N}},
$$

where $\Delta N$ denotes the signal loss in photon count due to photobleaching.

To obtain a sufficient SNR to recover the sample's features, in practice the differential intensity was averaged along the time course, yielding a final PIM image with an improved SNR

$$
S N R_{P I M} \approx \sqrt{M} S N R_{\text {diff }},
$$

where $M$ is the total number of acquired differential images. The microscope's focal drift compensation module can be employed to correct for the loss of focus caused by temperature changes around the microscope and other factors during time-lapse observation.

To extract high-order components from the PIM image, the time-lapse fluorescent decay at each pixel was fitted to a polynomial equation

$$
I_{f}(t)=c_{1}+c_{2} t+c_{3} t^{2}
$$

where $t$ is time, and $c_{1}, c_{2}$, and $c_{3}$ are the coefficients associated with $F, F^{2}$ and $F^{3}$, respectively.

\section{RESULTS AND DISCUSSION}

We first evaluated the lateral resolution of PIM by imaging a sharp edge of a thin fluorescent film (maximal emission wavelength $\sim 520 \mathrm{~nm}$ ) with a confocal fluorescence microscope. With an oil-immersion objective with a numerical aperture (NA) of 1.4, the full width at half maximum (FWHM) of the PSF measured by PIM was $\sim 110 \mathrm{~nm}$, less than

half of the $\sim 280 \mathrm{~nm}$ measured by conventional confocal microscopy (Fig. 1). 


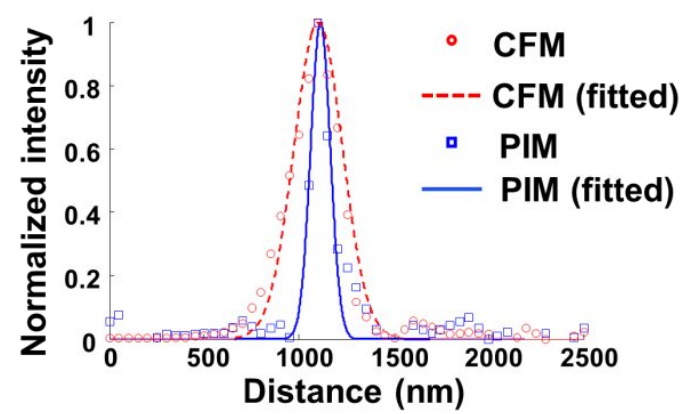

Fig. 1. Comparative measurement of the lateral resolutions of confocal fluorescence microscopy (CFM) and PIM with an oil-immersion microscope objective $(\mathrm{NA}=1.4)$. The fitted PSFs are shown by dashed and solid lines. The full width at half maximum (FWHM) of CFM's PSF is $\sim 280 \mathrm{~nm}$, while that of PIM's PSF is $\sim 110 \mathrm{~nm}$.

To demonstrate the sub-diffraction-limited imaging capability of PIM in a biological cell, we imaged cellular microtubules (bovine pulmonary artery endothelial cell line) stained with a green fluorescent dye, BODIPY FL Goat Anti-Mouse IgG (F-14781, Life Technologies). The fluorophore was excited and photobleached by an argon laser at 488 nm. Following a similar procedure, 200 time-lapse fluorescence images were measured to extrapolate the PIM image, shown in Fig. 2a. The signal intensities across a line in the zoomed area were plotted and compared between the confocal fluorescence and PIM images (Fig. 2b).

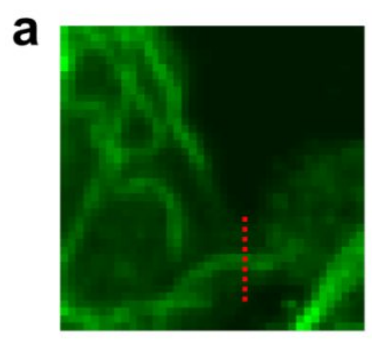

CFM

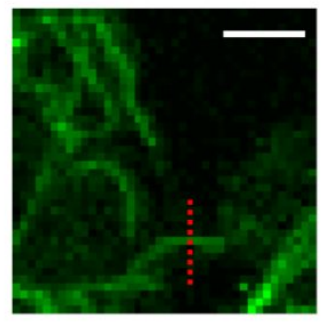

PIM

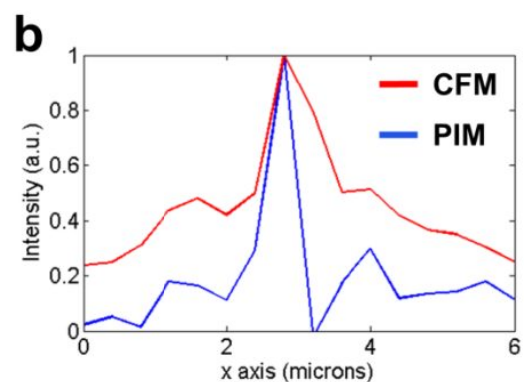

Fig. 2. Sub-diffraction-limited fluorescence imaging by PIM. a. Confocal fluorescence microscopy (CFM) and PIM images of cellular microtubules stained with a green fluorescent dye, BODIPY FL Goat Anti-Mouse IgG. Scale bar, 5 $\mu \mathrm{m}$. b. Comparison of fluorescent intensities along the dashed line in a.

In addition, we also imaged cellular mitochondria (NIH 3 T3 fibroblast cell line) stained with an orange fluorophore, Mitotracker CMT (M7510, Life Technologies). The sample was excited and photobleached by a He-Ne laser at $543 \mathrm{~nm}$. The acquired confocal fluorescence and PIM images are shown in Fig. 3. Since PIM relies on fluorescence decay to extract the nonlinear components, we evaluated the gain in resolution improvement against the loss of fluorescence intensity level after the PIM procedure. A measurement showed that the cellular fluorescent intensity was decreased by $\sim 10 \%$ due to photobleaching, indicating the feasibility of multi-cycle PIM imaging before the fluorophore loses its entire fluorescing capability. 

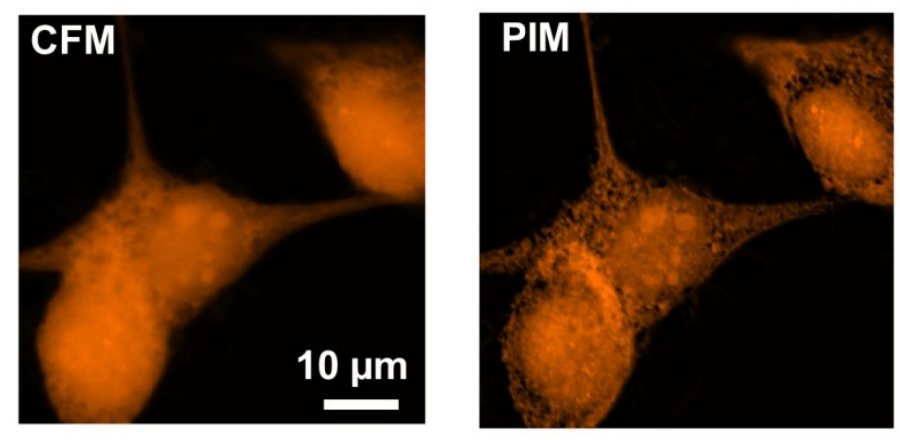

Fig. 3. Confocal fluorescence microscopy (CFM) and photobleaching imprinting microscopy (PIM) of cellular mitochondria stained with an orange fluorophore, Mitotracker CMT.

\section{CONCLUSION}

In summary, we presented a generic sub-diffraction-limited technique, photobleaching imprinting microscopy, for biological fluorescence imaging. In cellular imaging experiments, PIM demonstrated over a two-fold improvement in resolution. Compared with other super-resolution imaging techniques, PIM is easier to implement on a standard microscope with generic fluorescent dyes, making super-resolution imaging a routine lab technique. It is worth noting that, although techniques such as deconvolution microscopy can also remove image blur, the accuracy is highly dependent on the signal-to-background ratio[14].

Although not demonstrated here, PIM can be potentially employed in wide-field fluorescence microscopy [15] and light-sheet fluorescence microscopy (LSFM) [16] as well. Due to a structured fluence distribution along the depth axis, on the one hand, wide-field fluorescence PIM can be used to remove the out-of-focus light, thereby allowing for optical sectioning; on the other hand, light-sheet fluorescence PIM can be exploited to further improve axial resolution, offering a simple solution to a long-standing problem in LSFM on mitigating the trade-off between imaging field-of-view and sectioning thickness [17].

The resolution improvement by PIM is achieved at the expense of fluorescence loss. A $10 \%$ drop in fluorescence intensity was observed after the PIM procedures. In addition, averaging the differential intensity along the fluorescence decay course is normally required to obtain a sufficient SNR to extrapolate the high-order components, a process that decreases the temporal resolution of the microscope and currently allows monitoring of only slow-varying events. This limitation can be potentially overcome by choosing a smaller FOV, scanning a path of interest with random access, or shortening the pixel dwell time using a higher fluorophore concentration. Moreover, parallel acquisition schemes, such as spinning-disk confocal microscopy, can also be employed. Since PIM is based on the photobleaching memory effect, the achievable resolution is dependent on the light fluence distribution on the focal plane. In deep imaging, the focal diameter deviates from the diffraction limit due to light scattering [18], a fact that degrades PIM's resolution as well. Consequently, in practice, the imaging depth of PIM is still restricted by the optical diffusion limit, which is approximately $1 \mathrm{~mm}$ in biological tissue.

For convenient operation, the data acquisition in the presented work was conducted in a frame-based manner. At each sampling pixel, the time interval between two consecutive excitation events is limited by the frame acquisition time, which can be relatively long due to a large number of spatial sampling pixels. This limitation may compromise the PIM image formation in live cultures because the phenomena such as fluorescence recovery after photobleaching (FRAP) can erase sample's memory on the previous light field distribution. To overcome this problem, the data acquisition of PIM can be switched to a point-based approach, measuring the entire fluorescence decay at a sampling pixel before the scanner moves to the next location. This method can minimize the time interval between two consecutive excitation events down to the detector's integration time (200 $\mu \mathrm{s}$ in the presented study), a time scale within which the diffusion of fluorophores can be generally neglected.

\section{REFERENCES}

1. J. W. Lichtman and J. A. Conchello, "Fluorescence microscopy," Nat Methods 2(12), 910-919 (2005) 
2. M. J. Rust, M. Bates and X. W. Zhuang, "Sub-diffraction-limit imaging by stochastic optical reconstruction microscopy (STORM)," Nat Methods 3(10), 793-795 (2006)

3. E. Betzig, G. H. Patterson, R. Sougrat, O. W. Lindwasser, S. Olenych, J. S. Bonifacino, M. W. Davidson, J. Lippincott-Schwartz and H. F. Hess, "Imaging intracellular fluorescent proteins at nanometer resolution," Science 313(5793), 1642-1645 (2006)

4. $\quad$ S. T. Hess, T. P. K. Girirajan and M. D. Mason, "Ultra-high resolution imaging by fluorescence photoactivation localization microscopy," Biophys J 91(11), 4258-4272 (2006)

5. $\quad$ S. Munck, K. Miskiewicz, R. Sannerud, S. A. Menchon, L. Jose, R. Heintzmann, P. Verstreken and W. Annaert, "Sub-diffraction imaging on standard microscopes through photobleaching microscopy with non-linear processing," $J$ Cell Sci 125(9), 2257-2266 (2012)

6. D. T. Burnette, P. Sengupta, Y. H. Dai, J. Lippincott-Schwartz and B. Kachar, "Bleaching/blinking assisted localization microscopy for superresolution imaging using standard fluorescent molecules," P Natl Acad Sci USA 108(52), 21081-21086 (2011)

7. P. D. Simonson, E. Rothenberg and P. R. Selvin, "Single-Molecule-Based Super-Resolution Images in the Presence of Multiple Fluorophores," Nano Lett 11(11), 5090-5096 (2011)

$8 . \quad$ T. A. Klar, S. Jakobs, M. Dyba, A. Egner and S. W. Hell, "Fluorescence microscopy with diffraction resolution barrier broken by stimulated emission," P Natl Acad Sci USA 97(15), 8206-8210 (2000)

9. M. G. L. Gustafsson, "Surpassing the lateral resolution limit by a factor of two using structured illumination microscopy," J Microsc-Oxford 198, 82-87 (2000)

10. M. G. L. Gustafsson, "Nonlinear structured-illumination microscopy: Wide-field fluorescence imaging with theoretically unlimited resolution," P Natl Acad Sci USA 102(37), 13081-13086 (2005)

11. $\quad$ S. W. Hell, "Far-field optical nanoscopy," Science 316(5828), 1153-1158 (2007)

12. R. Y. Tsien, L. Ernst and A. Waggoner, "Fluorophores for Confocal Microscopy: Photophysics and Photochemistry," in Handbook Of Biological Confocal Microscopy J. B. Pawley, Ed., Springer (2006).

13. G. H. Patterson and D. W. Piston, "Photobleaching in two-photon excitation microscopy," Biophys J 78(4), 2159-2162 (2000)

14. J. M. Murray, "Methods for imaging thick specimens: confocal microscopy, deconvolution, and structured illumination," Cold Spring Harb Protoc 2011(12), 1399-1437 (2011)

15. C. Y. Li, L. Gao, Y. Liu and L. H. V. Wang, "Optical sectioning by wide-field photobleaching imprinting microscopy," Appl Phys Lett 103(18), 183703 (2013)

16. L. Gao, L. Zhu, C. Li and L. V. Wang, "Nonlinear light-sheet fluorescence microscopy by photobleaching imprinting," Journal of The Royal Society Interface 11(93), 20130851 (2014)

17. F. O. Fahrbach, V. Gurchenkov, K. Alessandri, P. Nassoy and A. Rohrbach, "Self-reconstructing sectioned Bessel beams offer submicron optical sectioning for large fields of view in light-sheet microscopy," Opt. Express 21(9), 11425-11440 (2013)

18. Y. Liu, C. Zhang and L. V. Wang, "Effects of light scattering on optical-resolution photoacoustic microscopy," J Biomed Opt 17(12), 126014-126014 (2012) 